\title{
Clinical results of Trabectome surgery for open-angle glaucoma
}

This article was published in the following Dove Press journal:

Clinical Ophthalmology

9 October 2015

Number of times this article has been viewed

\section{Takanori Mizoguchi' \\ Shiro Nishigaki \\ Tomoki Sato ${ }^{3}$ \\ Harumi Wakiyama ${ }^{4}$ \\ Nobuchika Ogino ${ }^{2}$}

'Mizoguchi Eye Clinic, Ophthalmology, Sasebo, ${ }^{2}$ Nishigaki Eye Clinic,

Ophthalmology, Nagoya, ${ }^{3}$ Sato Eye

Clinic, Ophthalmology, Arao, ${ }^{4}$ Japanese

Red Cross Nagasaki Genbaku

Hospital, Nagasaki, Japan
Correspondence: Takanori Mizoguchi Mizoguchi Eye Clinic, 6-13 Tawaramachi, Sasebo, Nagasaki 857-00I6, Japan

$\mathrm{Tel}+81956225681$

Fax +81956256040

Email t-mizo@siren.ocn.ne.jp
Background: The purpose of this study was to determine outcomes when using Trabectome surgery and to evaluate factors associated with its effects in primary open-angle glaucoma (POAG) and exfoliation glaucoma (EXG).

Methods: This was a prospective, non-randomized, observational, comparative cohort study in which Trabectome surgery was used alone in patients with POAG or EXG. Trabectome surgery was considered to have failed when at least one of the following three criteria was fulfilled: intraocular pressure (IOP) $\geq 21 \mathrm{mmHg}$ and a $<20 \%$ reduction below the baseline IOP on two consecutive follow-up visits 3 months or more after surgery; need for additional glaucoma surgery; and an increase in number of medications compared with baseline.

Results: The subjects were 32 males (34 eyes) and 46 females (48 eyes). POAG was observed in 43 eyes and EXG in 39 eyes. IOP after Trabectome surgery decreased significantly from $22.3 \pm 6.8 \mathrm{mmHg}$ at baseline to $14.0 \pm 3.9 \mathrm{mmHg}$ (23.0\% reduction) at month 24 in all cases $(P<0.0000)$. The success rate at 2 years was $51.2 \%$ for all cases (POAG, 50.9\%; EXG, 49.2\%). There was no significant difference in success rate between POAG and EXG $(P=0.91)$. Preoperative IOP $(P=0.033)$ and number of medications $(P=0.041)$ were significant factors for surgical success/failure in multivariate logistic regression. No serious complications were observed.

Conclusion: Trabectome surgery achieved favorable IOP control and was equally effective in patients with POAG and those with EXG. Its effects were influenced by preoperative IOP and number of preoperative medications.

Keywords: Trabectome, primary open-angle glaucoma, exfoliation glaucoma, success rate, risk factors

\section{Introduction}

Trabeculectomy with adjunctive mitomycin $\mathrm{C}$ is considered to be the gold standard procedure for lowering intraocular pressure (IOP) in patients with glaucoma, but is frequently accompanied by short-term and long-term complications. ${ }^{1}$ These complications are especially problematic in the treatment of glaucoma. ${ }^{2-4}$

The Trabectome (Neomedix Corporation, Tustin, CA, USA) was developed as a minimal invasive surgical device for open-angle glaucoma. In open-angle glaucoma, the inner wall of Schlemm's canal and the juxtacanalicular trabecular meshwork show resistance to aqueous humor outflow. ${ }^{5}$ Ablation of these areas using the Trabectome without damaging the collector channel facilitates aqueous outflow, reducing the IOP. This surgical method is minimally invasive because it can be performed after a corneal incision, and the primary sites of abnormal flow resistance can be removed without damaging the conjunctiva. ${ }^{6}$

A multi-institutional study showed favorable results of Trabectome surgery, suggesting that this procedure is safe, minimally invasive, and associated with few complications. ${ }^{6,7}$ In addition, Trabectome surgery performed simultaneously with 
cataract surgery has produced favorable results, and cataract complicated by glaucoma has been regarded as a good indication for simultaneous surgery. ${ }^{6-8}$ However, a recent study of Trabectome surgery at two institutions by Ahuja et $\mathrm{al}^{9}$ and Jea et $\mathrm{al}^{10}$ using the Kaplan-Meier curve showed low 2-year success rates $(22.4 \%$ and $39.2 \%$, respectively). In a study by Minckler et $\mathrm{al}^{6}$ Trabectome surgery was performed by many surgeons in multiple institutions, so the details of patients who could be followed up were unknown. Studies performed by one surgeon at one institution in which the courses of the subjects are strictly followed may be clinically valuable. ${ }^{9,10}$

Although this study was performed at two institutions, one surgeon in each institution performed the surgery. The subjects could be followed up closely. The results of Trabectome surgery were evaluated in these subjects, and risk factors affecting the results were identified.

\section{Methods}

This was a prospective case-control study and included patients with open-angle glaucoma (OAG) who visited the eye clinics at Mizoguchi and Nishigaki between April 1, 2011 and September 30, 2013. The study followed the tenets of the Declaration of Helsinki and had the approval of the ethics committee of each participating facility. Written informed consent was obtained from each patient before enrollment in the study. The inclusion criteria were age $\geq 20$ years with primary open-angle glaucoma (POAG) or exfoliation glaucoma (EXG). Eyes that presented with a baseline IOP $\geq 21 \mathrm{mmHg}$ while on ocular hypotensive medications before surgery were included. Eyes that presented with an IOP $<21 \mathrm{mmHg}$ while on ocular hypotensive medications before surgery in which target pressure ( $\geq 20 \%$ reduction from baseline IOP with medications) could not be achieved were also included. In order to evaluate the outcomes in eyes with POAG versus EXG after Trabectome surgery, we performed a subanalysis by dividing them into two subgroups based on type of open-angle glaucoma (ie, POAG or EXG). Trabectome patients who had undergone cataract, glaucoma, or vitreous surgery without complications 1 year or more earlier were also included. Patients who underwent simultaneous cataract and Trabectome surgery or had closed-angle or glaucoma secondary to uveitis were excluded.

Visual acuity, IOP, and number of medications were recorded at the time of the postoperative visit. The preoperative (baseline) IOP was the mean of the IOPs recorded at two visits while on preoperative treatment. The IOP was measured at 10 am using Goldmann applanation tonometry. Concerning the number of medications, each type of medication was counted as 1 , and each fixed combination was counted as 2 .

Success was defined as a postoperative IOP $<21 \mathrm{mmHg}$ and $\mathrm{a} \geq 20 \%$ reduction from baseline. Cases were considered as treatment failures if the success criterion was not met at two consecutive visits after 3 postoperative months. Treatment failure also included an increase in glaucoma medications from baseline at two consecutive visits after 3 postoperative months and need for additional glaucoma surgery.

\section{Trabectome procedure}

A $1.7 \mathrm{~mm}$ keratome was used to create a beveled temporal clear corneal incision. The head and microscope were tilted. The surgical goniolens were placed on the cornea and nasal trabecular meshwork was visualized with surgical goniolens. The anterior chamber was filled with viscoelastic to facilitate insertion of Trabectome hand piece. The probe was used to ablate between 90 and 120 degrees of the nasal trabecular meshwork and inner wall of Schlemm's canal. After a single 10-0 nylon suture was placed, the viscoelastic material was completely removed and replaced with balanced salt solution (Alcon, Fort Worth, TX, USA).

Postoperatively, all patients were treated with levofloxacin hydrate $1.5 \%$ four times per day, fluorometholone $0.1 \%$ four times per day, and $2 \%$ pilocarpine four times per day for 3 weeks, which was tapered over the next 1-3 weeks depending on the level of anterior chamber response, and anti-glaucoma medications were added if needed.

\section{Statistical analysis}

All analyses were carried out using $\mathrm{JMP}^{\circledR} 8$ (SAS Institute Inc., Cary, NC, USA). Baseline and postoperative values were compared by a one-way repeated-measures analysis of variance (ANOVA) test for each group. The difference in IOP and number of antiglaucoma medications between POAG and EXG were assessed by an unpaired $t$-test. Pearson's $\chi^{2}$ test was used for subgroup comparison of sex and lens status before surgery. Kaplan-Meier survival curves were constructed and then compared using the log-rank test. Univariate logistic regression and multivariate logistic regression analyses were performed to determine factors associated with failure of Trabectome surgery using the criteria. A $P$-value less than 0.05 was considered to be statistically significant.

\section{Results}

\section{Baseline characteristics}

The baseline characteristics of all cases and subgroup cases are shown in Table 1. The subjects comprised 78 patients 
Table I Baseline demographics for all cases and subgroups of cases

\begin{tabular}{|c|c|c|c|c|}
\hline & \multirow[t]{2}{*}{ All cases } & \multicolumn{2}{|l|}{ Subgroups } & \multirow[t]{2}{*}{$P$-value } \\
\hline & & POAG & EXG & \\
\hline Patients, number of eyes, (\%) & $78(82)$ & $39(43)$ & $39(39)$ & \\
\hline Age, years, mean $\pm S D$ & $73.3 \pm 13.0$ & $67.4 \pm 14.1$ & $79.7 \pm 7.5$ & $<0.0001$ \\
\hline \multicolumn{5}{|l|}{ Sex, n } \\
\hline Female & 46 (48 eyes) & 24 (27 eyes) & 22 ( 22 eyes) & \\
\hline Male & 32 (34 eyes) & 15 (16 eyes) & 17 (17 eyes) & 0.56 \\
\hline \multicolumn{5}{|l|}{ Lens status, number of eyes, (\%) } \\
\hline Phakic & $19(23.2)$ & $12(27.9)$ & $7(17.9)$ & \\
\hline Pseudophakic & $63(96.8)$ & $31(72.1)$ & $32(82.1)$ & 0.29 \\
\hline Mean deviation $(\mathrm{dB})$, mean $\pm \mathrm{SD}$ & $-13.9 \pm 9.2$ & $-13.4 \pm 9.0$ & $-14.4 \pm 9.5$ & 0.62 \\
\hline \multicolumn{5}{|c|}{ Prior treatment, number of eyes, (\%) } \\
\hline Argon laser trabeculoplasty & 0 & 0 & 0 & \\
\hline Selective laser trabeculoplasty & 0 & 0 & 0 & \\
\hline Trabeculotomy & $17(20.7)$ & $5(I 1.6)$ & $12(30.8)$ & \\
\hline Trabeculectomy & $4(4.9)$ & $3(7.0)$ & I (2.6) & \\
\hline Pars plana vitrectomy & $2(2.4)$ & I (2.3) & I (2.6) & \\
\hline None & $59(72.0)$ & 34 (79.1) & $25(64.1)$ & \\
\hline
\end{tabular}

Abbreviations: POAG, primary open-angle glaucoma; EXG, exfoliation glaucoma; SD, standard deviation.

( 82 eyes). The subjects consisted of 32 males ( 34 eyes) and 46 females (48 eyes), and the mean ( \pm standard deviation) age at the time of surgery, baseline IOP, mean baseline number of medications, baseline mean deviation, and mean duration of follow-up period were $73.3 \pm 13.0$ years, $22.3 \pm 6.8 \mathrm{mmHg}$, $2.8 \pm 0.8,-13.9 \pm 9.2 \mathrm{~dB}$, and $19.0 \pm 7.1$ months, respectively.

\section{Postoperative results}

The postoperative course of IOP and number of medications are shown in Tables 2 and 3 , respectively. IOPs at 1 and 2 years after surgery were $15.1 \pm 3.2 \mathrm{mmHg}$ and $14.0 \pm 3.9 \mathrm{mmHg}$, respectively, showing significant decreases compared with baseline IOP $(P<0.0000$, ANOVA). There was a statistical significant difference in postoperative IOP at each follow-up

Table 2 Mean intraocular pressure of the operated eye before and after undergoing Trabectome surgery

\begin{tabular}{|c|c|c|c|c|}
\hline & \multirow{2}{*}{$\begin{array}{l}\text { All cases } \\
\text { (eyes) }\end{array}$} & \multicolumn{2}{|c|}{ Subgroup (eyes) } & \multirow[t]{2}{*}{$P$-value } \\
\hline & & POAG & EXG & \\
\hline Baseline & $22.3 \pm 6.8(82)$ & $23.5 \pm 7.2(43)$ & $21.7 \pm 6.2(39)$ & 0.22 \\
\hline \multicolumn{5}{|c|}{ Postoperatine visit } \\
\hline 6 months & $15.4 \pm 3.3(74)$ & $16.2 \pm 3.4(37)$ & $14.5 \pm 3.0(37)$ & 0.02 \\
\hline$\%$ reduction & $25.7 \pm 19.0$ & $23.8 \pm 15.7$ & $27.6 \pm 21.8$ & 0.40 \\
\hline 12 months & $\mid 5 . I \pm 3.2(60)$ & $15.7 \pm 3.0(29)$ & $\mid 4.6 \pm 3.4(3 \mid)$ & 0.22 \\
\hline$\%$ reduction & $24.0 \pm 19.5$ & $22.6 \pm 16.8$ & $24.4 \pm 22.1$ & 0.72 \\
\hline 18 months & $14.4 \pm 3.3(43)$ & $15.3 \pm 2.4(23)$ & $13.3 \pm 3.9(20)$ & 0.03 \\
\hline$\%$ reduction & $25.3 \pm 23.6$ & $22.7 \pm 20.0$ & $28.3 \pm 27.6$ & 0.44 \\
\hline 24 months & $14.0 \pm 3.9(22)$ & $\mid 4.1 \pm 2.2(8)$ & $13.9 \pm 4.7(14)$ & 0.89 \\
\hline$\%$ reduction & $23.0 \pm 25.3$ & $23.8 \pm 20.2$ & $21.0 \pm 28.4$ & 0.80 \\
\hline
\end{tabular}

Notes: Data are presented as mean \pm standard deviation. The values in the parenthesis refer to the the number of the eyes.

Abbreviations: POAG, primary open-angle glaucoma; EXG, exfoliation glaucoma. visit for POAG and EXG when compared with baseline IOP (both $P<0.0000$, ANOVA). Numbers of medications, 1 and 2 years after surgery, were $2.7 \pm 0.8$ and $2.6 \pm 0.8$, respectively, for all patients, showing no significant differences when compared with baseline ( $P=0.73$, ANOVA). A significant decrease in antiglaucoma medications was observed only at 24 months after surgery in POAG ( $P=0.016$, ANOVA). However, there was no significant difference in the number of antiglaucoma medications at each time point in patients with EXG compared with baseline ( $P=0.53$, ANOVA). A significant difference in number of antiglaucoma medications between POAG and EXG was observed only at 2 years after surgery ( $P=0.003$; Table 3$)$.

The success rate calculated using the Kaplan-Meier curve was $51.2 \%$ at 24 months after Trabectome surgery (Table 4 ). The success rate for POAG and EXG at 24 months after surgery was $50.9 \%$ and $49.2 \%$, respectively, with no significant difference ( $P=0.91, \log$-rank test).

Table 3 Mean number of glaucoma medications in the operative eye before and after Trabectome surgery

\begin{tabular}{lllll}
\hline & $\begin{array}{l}\text { All cases } \\
\text { (eyes) }\end{array}$ & \multicolumn{2}{l}{ Subgroup (eyes) } & \multirow{2}{*}{ P-value } \\
\cline { 3 - 4 } & POAG & EXG & \\
\hline Baseline & $2.8 \pm 0.8(82)$ & $2.8 \pm 0.8(43)$ & $2.7 \pm 0.8(39)$ & 0.15 \\
6 months & $2.6 \pm 0.8(75)$ & $2.5 \pm 0.9(37)$ & $2.6 \pm 0.8(38)$ & 0.63 \\
12 months & $2.7 \pm 0.8(62)$ & $2.4 \pm 0.8(29)$ & $2.8 \pm 0.9(33)$ & 0.1 \\
18 months & $2.7 \pm 0.8(46)$ & $2.5 \pm 0.7(23)$ & $2.7 \pm 1.0(23)$ & 0.39 \\
24 months & $2.6 \pm 0.8(24)$ & $1.8 \pm 1.0(8)^{*}$ & $2.9 \pm 0.7(16)$ & 0.003 \\
\hline
\end{tabular}

Notes: *Significant decrease in antiglaucoma medications versus baseline in patients with POAG. Data are presented as mean \pm standard deviation. The values in the parenthesis refer to the the number of the eyes.

Abbreviations: POAG, primary open-angle glaucoma; EXG, exfoliation glaucoma. 
Table 4 Kaplan-Meier success rate (\%) for cases of Trabectome surgery

\begin{tabular}{lllll}
\hline & All cases (\%) & Subgroup & P-value \\
\cline { 3 - 4 } & & POAG (\%) & EXG (\%) & \\
\hline 6 months & 76.8 & 76.7 & 77.0 & \\
I2 months & 64.1 & 59.4 & 69.2 & \\
18 months & 53.6 & 59.4 & 49.2 & \\
24 months & 51.2 & 50.9 & 49.2 & 0.91 \\
\hline
\end{tabular}

Abbreviations: POAG, primary open-angle glaucoma; EXG, exfoliation glaucoma.

Postoperative complications are shown in Table 5. Early IOP spikes, defined as IOPs $>10 \mathrm{mmHg}$ above baseline within 1 week after surgery, occurred in four eyes (4.9\%). Hyphema was observed in 19 eyes (23.2\%) and microhyphema in the other 63 eyes (76.8\%). Hyphema was minimal and disappeared without any special management 1-10 days after surgery. At the time of the final examination, visual acuity had decreased by more than two lines when compared with preoperative visual acuity in one eye $(1.2 \%)$ because of cataract progression.

Of the eyes showing surgical failure, nine (11.0\%) required additional medications when compared with the number used at baseline due to a poor decrease in postoperative IOP, and 12 eyes (14.6\%) required additional glaucoma surgery at an average of 280 (range 8-796) days after initial surgery. Trabeculectomy with mitomycin $\mathrm{C}$ was performed in ten $(12.2 \%)$ eyes (seven eyes with POAG; three eyes with EXG), and trabeculotomy combined with deep sclerectomy without mitomycin $\mathrm{C}$ in two (2.4\%) eyes (one eye with POAG and the other with EXG). None of the patients underwent laser trabeculoplasty. Aggravation of cataract was observed in one eye after surgery.

Factors associated with failure of Trabectome surgery were evaluated by univariate logistic regression and multivariate logistic regression. On univariate logistic regression, surgical failure was significantly correlated with baseline IOP and a history of surgery, but not with sex, age, disease type, preoperative mean deviation, lens status, or number of

Table 5 Type and prevalence of postoperative complications following Trabectome surgery

\begin{tabular}{llllll}
\hline Complication & $\begin{array}{l}\text { All cases, number } \\
\text { of eyes (\%) }\end{array}$ & \multicolumn{2}{l}{\begin{tabular}{l} 
Sub-group \\
\cline { 3 - 4 }
\end{tabular}} & POAG & P-value \\
\hline Hyphema & $19(23.2)$ & 14 & 5 & 0.03 \\
Microhyphema & $63(76.8)$ & 29 & 34 & 0.03 \\
IOP spike $>10 \mathrm{mmHg}$ & $4(4.9)$ & $\mathrm{I}$ & 3 & 0.26 \\
VA decrease $>2$ lines & $\mathrm{I}(\mathrm{I} .2)$ & $\mathrm{I}$ & 0 & 0.34 \\
Cataract progression & $\mathrm{I}(\mathrm{I} .2)$ & $\mathrm{I}$ & 0 & 0.34 \\
\hline
\end{tabular}

Abbreviations: IOP, intraocular pressure; VA, visual acuity; POAG, primary openangle glaucoma; EXG, exfoliation glaucoma. medications at baseline (Table 6). As a result of selection of variables for multivariate logistic regression, the baseline IOP and baseline number of medications were left in the final model, and were significantly correlated with the success or failure of surgery (Table 6). The results of Trabectome surgery differed according to baseline IOP and number of antiglaucoma medications.

\section{Discussion}

Trabectome surgery significantly reduced the postoperative IOP in our study patients. Although the number of medications could not be significantly reduced after surgery, IOPs at 1 and 2 years after surgery were low $(15.1 \mathrm{mmHg}$ and $14.0 \mathrm{mmHg}$, respectively). These results suggest that Trabectome surgery can control IOP at a clinically useful level. IOPs, 1 and 2 years after Trabectome surgery, were reported to be $16.1 \mathrm{mmHg}$ and $16.6 \mathrm{mmHg}$, respectively, by Minckler et al, ${ }^{6} 17.4 \mathrm{mmHg}$ and $15.9 \mathrm{mmHg}$, respectively, by Jea et al, ${ }^{10}$ and $16.6 \mathrm{mmHg}$ and $16.8 \mathrm{mmHg}$, respectively, by Ahuja et al. ${ }^{9}$ Maeda et al ${ }^{11}$ reported an IOP of $17.9 \mathrm{mmHg}$ 1 year after surgery. Thus, a postoperative IOP in the high teens was frequently reported. In our patients, the postoperative IOP was in the mid teens, which is lower than that previously reported. ${ }^{6,9-11}$ However, in this study, the number of medications could not be significantly reduced after Trabectome surgery in all patients. Many studies have shown a significant decrease in number of medications after Trabectome surgery. ${ }^{6-12}$ In our study, the absence of a postoperative decrease in number of medications may have been partly because many patients had advanced glaucoma, with a mean deviation of the preoperative visual field of $-13.9 \mathrm{~dB}$ (median $-12.3 \mathrm{~dB}$ ), and the postoperative IOP needed to be maintained as low as possible. ${ }^{13}$

There was no significant difference in percent IOP reduction from baseline between POAG and EXG. However, there was a statistically significant difference in number of medications only at 24 months after surgery between POAG and EXG. This is very interesting. We think that the exfoliation material in the anterior wall of Schlemm's canal may have been accumulating and might have disturbed the ease of aqueous outflow for a long time after surgery.

The success rate using the Kaplan-Meier method after Trabectome surgery was reported to be $60 \%-65 \%$ after 2 years by Minckler et $\mathrm{al}^{6}$ and $62.9 \%-79.1 \%$ after 1 year by Ting et al. ${ }^{12}$ However, it was reported to be $22.4 \%$ after 2 years by Jea et $\mathrm{al}^{10}$ and $35.2 \%$ after 2 years by Ahuja et al. ${ }^{9}$ These authors suggested that the success rate was low due to strict follow-up because the study was performed in a single institution, and there were only a few dropouts. 
Table 6 Univariate logistic regression and multivariate logistic regression of failed Trabectome surgery in the treatment of open-angle glaucoma

\begin{tabular}{|c|c|c|c|c|c|c|c|c|c|}
\hline \multirow[t]{2}{*}{ Parameter } & \multirow[t]{2}{*}{ Category } & \multirow[t]{2}{*}{$\begin{array}{l}\text { Number } \\
\text { of eyes }\end{array}$} & \multirow{2}{*}{$\begin{array}{l}\text { Failure eye, } \\
\text { number of } \\
\text { eyes (\%) }\end{array}$} & \multicolumn{3}{|c|}{ Univariate logistic regression } & \multicolumn{3}{|c|}{$\begin{array}{l}\text { Multivariate logistic regression } \\
\text { (Backward elimination: } P<0.05 \text { ) }\end{array}$} \\
\hline & & & & OR & $95 \% \mathrm{Cl}$ & $P$-value & OR & $95 \% \mathrm{Cl}$ & $P$-value \\
\hline Eye & & 82 & $35(42.7)$ & & & & & & \\
\hline \multirow[t]{2}{*}{ Sex } & Male & 34 & $13(38.2)$ & Reference & & & & & \\
\hline & Female & 48 & $22(45.8)$ & 1.367 & $(0.559,3.345)$ & 0.494 & & & \\
\hline \multirow[t]{4}{*}{ Age, years } & $<68$ & 19 & $8(42.1)$ & Reference & & 0.886 & & & \\
\hline & $68 \leq \sim<77$ & 20 & $10(50.0)$ & 1.375 & $(0.388,4.867)$ & 0.621 & & & \\
\hline & $77 \leq \sim<82$ & 22 & $9(40.9)$ & 0.952 & $(0.274,3.308)$ & 0.938 & & & \\
\hline & $82 \leq$ & 21 & $8(38.1)$ & 0.846 & $(0.238,3.004)$ & 0.796 & & & \\
\hline \multirow{2}{*}{$\begin{array}{l}\text { Open-angle } \\
\text { glaucoma type }\end{array}$} & EXG & 39 & $16(4 \mid .0)$ & Reference & & & & & \\
\hline & POAG & 43 & $19(44.2)$ & 1.138 & $(0.473,2.736)$ & 0.773 & & & \\
\hline \multirow{2}{*}{$\begin{array}{l}\text { Prior glaucoma } \\
\text { surgery }\end{array}$} & $(-)$ & 57 & $20(35.1)$ & Reference & & & & & \\
\hline & $(+)$ & 25 & I5 (60.0) & 2.775 & $(1.055,7.302)$ & 0.039 & & & \\
\hline \multirow{5}{*}{$\begin{array}{l}\text { Mean } \\
\text { deviation, dB }\end{array}$} & $<-22.2$ & 19 & $9(47.4)$ & Reference & & 0.682 & & & \\
\hline & $-22.2 \leq \sim<-12.3$ & 19 & $7(36.8)$ & 0.648 & $(0.177,2.369)$ & 0.512 & & & \\
\hline & $-12.3 \leq \sim<-6.5$ & 18 & $9(50.0)$ & 1.111 & $(0.306,4.037)$ & 0.873 & & & \\
\hline & $\leq-6.5$ & 20 & $9(45.0)$ & 0.909 & $(0.258,3.204)$ & 0.882 & & & \\
\hline & None & 6 & I (I6.7) & 0.222 & $(0.022,2.280)$ & 0.205 & & & \\
\hline \multirow[t]{2}{*}{ Lens status } & Pseudophakic & 63 & $26(4 \mid .3)$ & Reference & & & & & \\
\hline & Phakic & 19 & $9(47.4)$ & 1.281 & $(0.457,3.590)$ & 0.638 & & & \\
\hline \multirow{4}{*}{$\begin{array}{l}\text { Baseline IOP, } \\
\mathrm{mmHg}\end{array}$} & $<18$ & 17 & $12(70.6)$ & Reference & & 0.042 & Reference & & 0.026 \\
\hline & $18 \leq \sim<22$ & 22 & $5(22.7)$ & 0.123 & $(0.029,0.519)$ & 0.004 & 0.123 & $(0.029,0.519)$ & 0.004 \\
\hline & $22 \leq \sim<28$ & 21 & $9(42.9)$ & 0.313 & $(0.08 I, I .2 I I)$ & 0.092 & 0.284 & $(0.072,1.117)$ & 0.072 \\
\hline & $28 \leq$ & 22 & $9(40.9)$ & 0.288 & $(0.075,1.108)$ & 0.070 & 0.168 & $(0.039,0.732)$ & \\
\hline \multirow{2}{*}{$\begin{array}{l}\text { Baseline } \\
\text { medications (n) }\end{array}$} & I, 2, 3 & 76 & $30(39.5)$ & Reference & & & Reference & & 0.019 \\
\hline & 4,5 & 6 & $5(83.3)$ & 7.666 & $(0.853,68.887)$ & 0.069 & $1 \mathrm{I} .493$ & $(1.107,119.324)$ & 0.020 \\
\hline
\end{tabular}

Note: Failure eye was defined as treatment failure if the success criterion was not met at two consecutive visits after 3 postoperative months.

Abbreviations: IOP, intraocular pressure; OR, odds ratio; $\mathrm{Cl}$, confidence interval; POAG, primary open-angle glaucoma; EXG, exfoliation glaucoma.

In the present study, the success rate was $51.8 \%$, which is higher than that reported by Jea et $\mathrm{al}^{10}$ or Ahuja et $\mathrm{al}^{9}$ but lower than the rate reported by Minckler et $\mathrm{al}^{6}$ and Ting et al. ${ }^{12}$ In our study, surgery was performed by one operator at each of the two institutions. In addition, both operators were glaucoma specialists who had performed many operations for glaucoma in daily practice, and postoperative follow-up could be performed with few dropouts in the two clinics. Thus, the evaluation method used in this study was similar to that used by Jea et al ${ }^{10}$ and Ahuja et al. ${ }^{9}$ The baseline IOP in our study was $22.7 \mathrm{mmHg}$, which is markedly lower than that in the studies by Minckler et $\mathrm{al}^{6}{ }^{6}(25.7$ $\mathrm{mmHg})$ or Jea et al ${ }^{10}(28.1 \mathrm{mmHg})$. This low baseline IOP may have affected our success rate. Trabectome surgery has been reported to reduce the IOP by about $20 \%-30 \%$, which is not a marked reduction. According to the relevant studies, ${ }^{6-12}$ the success rate after Trabectome surgery may be about $30 \%-50 \%$.
In our study, multivariate logistic regression showed a significant correlation between baseline IOP, number of medications, and the results of Trabectome. The Trabectome failure rate was high in eyes with a baseline IOP $<18 \mathrm{mmHg}$, low in those with a baseline IOP of 18-22 $\mathrm{mmHg}$, and high in those with a baseline IOP $\geq 8 \mathrm{mmHg}$. Thus, the effects of Trabectome surgery differed according to baseline IOP. Although the association of the baseline IOP level with surgical failure/success is unclear, a low or markedly high baseline IOP may be associated with surgical failure. Ahuja et $\mathrm{al}^{9}$ and Jea et $\mathrm{al}^{10}$ also reported that high and low baseline IOPs are risk factors for poor surgical outcomes. Thus, surgical results may differ according to baseline IOP.

Several studies have shown favorable results of Trabectome surgery in patients with EXG when compared with POAG. ${ }^{9}, 11,12$ However, the reasons for this difference are unclear. As a possible explanation, there was a report that the pathology in POAG is localized to the trabecular meshwork 
and distal outflow system, ${ }^{5}$ whereas the pathology in EXG is localized only to the trabecular meshwork. ${ }^{14}$ However, in our study, the surgical results did not differ between POAG and EXG. Exfoliation syndrome is an age-related disorder characterized by abnormal accumulation of extracellular elastin-related microfibrillar material throughout the anterior segment, especially in the inner wall of the trabecular meshwork. ${ }^{15}$ Thus, aging may be one of the most important factors for surgical failure in patients with EXG. In this study, cases with EXG were older than those with POAG, and included advanced cases. This is the possibility that the results for the treatment of EXG had be getting worse compared with several studies..$^{9,11,12}$ Nevertheless, the pathophysiology of treatment failure remains unknown.

Concerning the advantages, Trabectome surgery is minimally invasive and associated with a low incidence of postoperative complications, with no serious complications. The complication requiring the closest attention is postoperative IOP spikes. In our study, early IOP spikes were observed in four eyes (4.9\%), of which only one showed an IOP of $45 \mathrm{mmHg}$, and the other three showed high IOPs (25-28 mmHg). The high IOPs improved soon after drug administration. Only one eye $(1.2 \%)$ showed a decrease in visual acuity by more than two lines after surgery. Therefore, Trabectome surgery is safe, as previously reported. ${ }^{6-12}$

The limitations of this study are as follows. First, there was a possibility of bias in the selection of the Trabectome versus other surgical techniques for glaucoma. However, exclusion of bias may be difficult at present as its clinical usefulness is still unclear. Further prospective studies are necessary. The second limitation is the possibility that surgical manipulation differed between the two surgeons in the two institutions. However, in the study by Minckler et $\mathrm{al}^{6}$ more surgeons in multiple institutions performed Trabectome surgery, and so the possibility of bias in the evaluation of surgical effects may have been greater.

In conclusion, Trabectome surgery significantly reduced the postoperative IOP, but its effects differed according to the baseline IOP, and could not reduce the number of medications after surgery. However, the postoperative IOP was in the

Clinical Ophthalmology

\section{Publish your work in this journal}

Clinical Ophthalmology is an international, peer-reviewed journal covering all subspecialties within ophthalmology. Key topics include: Optometry; Visual science; Pharmacology and drug therapy in eye diseases; Basic Sciences; Primary and Secondary eye care; Patient Safety and Quality of Care Improvements. This journal is indexed on Submit your manuscript here: http://www.dovepress.com/clinical-ophthalmology-journal mid teens, being lower than the levels previously reported. Trabectome surgery is associated with few complications, does not disturb the conjunctiva, and is worth performing in patients with early-stage glaucoma.

\section{Disclosure}

The authors report no conflicts of interest in this work.

\section{References}

1. Rulli E, Biagioli E, Riva I, et al. Efficacy and safety of Trabeculectomy vs nonpenetrating surgical procedures. A systematic review and metaanalysis. JAMA Ophthalmol. 2013;131:1573-1582.

2. Bindish R, Condon GP, Schlosser JD, D'Antonio J, Lauer KB, Lehrer R. Efficacy and safety of mitomycin-C in primary trabeculectomy: fiveyear follow-up. Ophthalmology. 2002;109:1336-1341.

3. Soltau JB, Rothman RF, Budenz DL, et al. Risk factors for glaucoma filtering bleb infections. Arch Ophthalmol. 2000;118:338-342.

4. DeBry PW, Perkins TW, Heatley G, Kaufman P, Brumback LC. Incidence of late-onset bleb-related complications following trabeculectomy with mitomycin C. Arch Ophthalmol. 2002;120:297-300.

5. Johnson M. What controls aqueous humour outflow resistance? Exp Eye Res. 2006;82:545-557.

6. Minckler DM, Mosaed S, Dustin L, Francis B; the Trabectome Study Group. Trabectome (trabeculectomy-internal approach): addition experience and extended follow-up. Trans Am Ophthalmol Soc. 2008;106:149-160.

7. Francis B, Minckler D, Dustin L, et al. Combined cataract extraction and trabeculotomy by the internal approach for coexisting cataract and open-angle glaucoma: initial results. J Cataract Refract Surg. 2008; 34:1096-1103.

8. Jordan JF, Wecker T, van Oterendorp C, et al. Trabectome surgery for primary open-angle glaucoma and secondary open-angle glaucomas. Graefes Arch Clin Exp Ophthalmol. 2013;251:2753-2760.

9. Ahuja Y, Ma Khin Pyi S, Malihi M, Hodge DO, Sit AJ. Clinical results of ab interno trabeculotomy using the trabectome for openangle glaucoma: The Mayo Clinic Series in Rochester, Minnesota. Am J Ophthalmol. 2013;156:927-935.

10. Jea SY, Francis BA, Vakili G, Filippopoulos T, Rhee DJ. Ab interno trabeculectomy versus trabeculectomy for open-angle glaucoma. Ophthalmology. 2012;119:36-42.

11. Maeda M, Watanabe M, Ichikawa K. Evaluation of trabectome in openangle glaucoma. J Glaucoma. 2013;22:205-208.

12. Ting JL, Damji KF, Stiles MC; Trabectome Study Group. Ab interno trabeculectomy: outcomes in exfoliation versus primary open-angle glaucoma. J Cataract Refract Surg. 2012;38:315-323.

13. [No authors listed]. The effectiveness of intraocular pressure reduction in the treatment of normal-tension glaucoma. The Collaborative Normal-Tension Glaucoma Study Group. Am J Ophthalmol. 1998; 126:498-505.

14. Tektas OY, Lutjen-Drecoll E. Structural changes of the trabecular meshwork in different kinds of glaucoma. Exp Eye Res. 2009;88: 769-775.

15. Ritch R, Schlotzet-Schrehardt. Exfoliation syndrome. Surv Ophthalmol. 2001;45:265-315.
PubMed Central and CAS, and is the official journal of The Society of Clinical Ophthalmology (SCO). The manuscript management system is completely online and includes a very quick and fair peer-review system, which is all easy to use. Visit http://www.dovepress.com/ testimonials.php to read real quotes from published authors. 